\title{
Geometry Optimization of Pulsed Inductors
}

\author{
S. BARTKEviČIUSS ${ }^{a}$, J. NOVICKIJ ${ }^{a}$ AND N. VIŠNIAKOV ${ }^{b}$ \\ ${ }^{a}$ Department of Electrical Engineering, Vilnius Gediminas Technical University \\ Naugarduko st. 41, LT-03227, Vilnius, Lithuania \\ ${ }^{b}$ Department of Mechanical Engineering, Vilnius Gediminas Technical University \\ J. Basanavičiaus st. 28, LT-03224, Vilnius, Lithuania
}

\begin{abstract}
The optimization of pulsed power inductors' geometry is a difficult multi-dimensional task. On the one hand, an inductor could be destroyed because of the Joule overheating, on the other hand, it might be damaged by the Lorentz forces acting in its windings. The magnetic field pulse up to $50 \mathrm{~T}$ is the target while fields of this magnitude are the interest in many scientific applications. All factors mentioned are directly dependent on inductor's geometry, i.e. on the number of winding layers and windings per layer. Therefore, a detailed analysis of thermodynamic, electromagnetic and mechanical processes is inevitable and was carried out for different geometrical inductor configurations to reach the goal: a non-destructive inductor that can meet required magnetic field parameters. Direct calculation methods were used to create universal algorithms in MATLAB environment. The zone of inductor geometries, where stresses do not exceed the yield strength of materials, inductor is not overheated and generated magnetic field magnitude is in acceptable range, is given as a result which enables to manufacture new prototypes saving additional costs and time.
\end{abstract}

PACS numbers: 07.55.Db, 84.32.Hh, 85.70.Ay

\section{Introduction}

It is well known that high magnetic fields are a powerful tool for scientific research. Apart from the promising new discoveries in previously uncharted territory, higher magnetic fields give better resolution and enhanced sensitivity to many experiments; therefore the quest for ever higher fields is an important facet of contemporary solid state research [1].

It is important to notice that generating constant high magnetic field in many cases is an expensive luxury because of facilities, materials and other resources needed. Therefore scientists often turn to low energy consumption pulsed technologies and pulsed magnetic fields that often are sufficient enough for carrying out most of the experiments where these fields have to be applied. A relatively simple system included a capacitor bank, a pulsed switch and an inductor can be used for pulsed magnetic field generation up to 60-70 $\mathrm{T}[2]$.

However, simple principle of operation requires great efforts to ensure longer operation of applied pulsed inductors. The design of non-destructive pulsed coils is a complex technical problem because they operate under very heavy conditions. Pulsed coil construction has to deal with mechanical, thermal and electrical overloads occurring due to the Lorentz force and Joule heating [3].

In this research approximate mathematical methods and calculations were applied to create and solve universal and flexible thermodynamic, electromagnetic and electromechanical inductor models that were used later to find the zone of inductor geometries that could be used for inductor multi-pulse operation. For this purpose the Matlab ${ }^{\circledR}$ Simulink ${ }^{\circledR}$, and Matlab ${ }^{\circledR}$ programming environment software was used and authors' software packages were written.

\section{Thermodynamic, electromagnetic and electromechanical inductor's modelling}

Inductor's thermodynamic model is the base for further magnetic and mechanical analysis because the result is the transient process of a current which flows in inductor windings during the pulse. The analysis of this transient process in time enables to calculate the winding heating. The maximum current amplitude gives the allowance to calculate the distribution of the magnetic field in the inductor bore and windings $[4,5]$. This enables to calculate the maximum stress distribution which is a product of magnetic field density and flowing current. The current density is considered to be constant in all inductor windings.

\section{Inductor geometry optimization}

First of all, basic inductor parameters and limitations for optimization must be selected. As it was decided, inductor is winded using one monolithic wire and the winding configuration is selected using results from previous experiments [5] and is given in Table. The winding cross-section is shown in Fig. 1. 
TABLE

Winding's parameters.

\begin{tabular}{c|c}
\hline \hline Parameter & Value \\
\hline material & $\mathrm{CuNb}$ \\
\hline dimensions & $4.2 \times 2.37 \mathrm{~mm}$ \\
\hline composition & $82 \% \mathrm{Cu}$ \\
\hline$\sigma[\%]$ IACS & $63(293 \mathrm{~K})$ \\
\hline winding & S2 glass $^{\circledR} 0.3 \mathrm{~mm}$ \\
\hline insulation & fiber $/$ Kapton $^{\circledR}, 0$ Epoxy, \\
interlayer & Zylon $^{\circledR}+$ Ep \\
reinforcement & $0.23 \mathrm{~mm}$
\end{tabular}

Insulations used for pulsed inductors lose their properties when kept in temperatures over $T_{\max }=600 \mathrm{~K}$, and the yield strength of the mechanically weakest element $\mathrm{CuNb}$ wire is $\sigma_{\mathrm{VM}}=850 \mathrm{MPa}$. Transient process duration of current pulse $t_{\mathrm{tp}}$ is also an important property because longer lasting pulse can cause more damage to the wire and its surrounding layers. Assuming this the criteria for inductor optimization is would be: $40 \mathrm{~T}<B_{z \max }<60 \mathrm{~T}, t_{\mathrm{tp}}<1 \mathrm{~ms}, T_{\max }<600 \mathrm{~K}$, $\sigma_{\mathrm{VM}}<800 \mathrm{MPa}$.

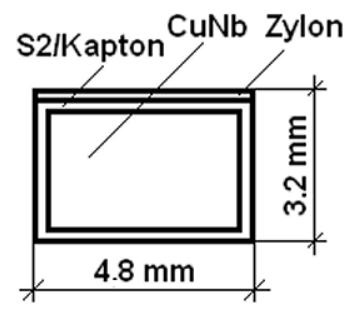

Fig. 1. Winding's cross-section.

Outer steel container and other possible reinforcements are not considered here because the displacement calculations are not performed. The criteria $\sigma_{\mathrm{VM}}$ is sufficient to determine whether $\mathrm{CuNb}$ fails. If maximum stress in inductor does not exceed the yield strength of $\mathrm{CuNb}$ wire, inductor will not fail mechanically.

Inputs for the optimization would be: winding number $n_{1}=\operatorname{var}=2: 20$; layer number $n_{2}=\operatorname{var}=2 ; 4 ; 6 ; 8$, bore radius $a_{1}=$ const. Following what was mentioned above, outputs would be: $B_{z}(\alpha, \beta), t_{\mathrm{tp}}(\alpha, \beta), T_{\max }(\alpha, \beta)$, $\sigma_{\mathrm{VM}}(\alpha, \beta)$, where $\alpha=a_{2} / a_{1}$ and $\beta=b / a_{1}, a_{2}$ being outer diameter of the coil and $b$ being half inductor length. The structure of a model for the optimization is shown in Fig. 2.

For the optimization and determination of inductor's "life-zone" the capacitor bank of total capacity of $C=$ $5000 \mu \mathrm{F}$ was always charged up to $U_{0}=3 \mathrm{kV}$, coil was cooled down to liquid nitrogen temperature $T_{0}=77 \mathrm{~K}$. The "life-zone" was found performing steps that are given in Sects. 2.2, 2.3, 2.4 while changing (increasing) $n_{1}$ and $n_{2}$.

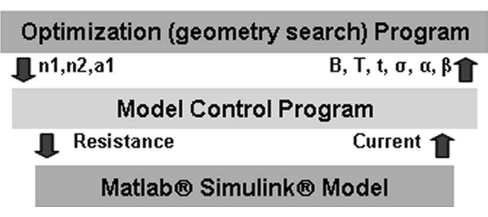

Fig. 2. Inductor optimization process structure.

\section{Results and discussion}

Axial magnetic field, transient process duration, maximum winding temperature and von Misses stress distributions over $\alpha$ and $\beta$ are shown in Fig. 3a-d, respectively. Hatched areas show inductor's geometries that do not fall into selected optimization criteria. Inductor's "life-zone" might be found while comparing these results. From Fig. 3a it is obvious that high magnetic fields usually used in experiments $\left(40 \mathrm{~T}<B_{z}<60 \mathrm{~T}\right)$ can be generated constructing 8-13 windings per layer and 2, 4, 6, 8 layer inductors. Figure 3b indicates that short pulses cannot be reached with high numbers of windings per layer and layers. This happens because of higher inductance $L_{\mathrm{I}}$. Longer pulse times are not applicable in some experiments while usually in these cases pulse maximum is reached very early $\left(20-30 \%\right.$ of the $\left.t_{\mathrm{tp}}\right)$ and the decay of the current lasts for longer which directly means higher winding temperatures at the end of the pulse.

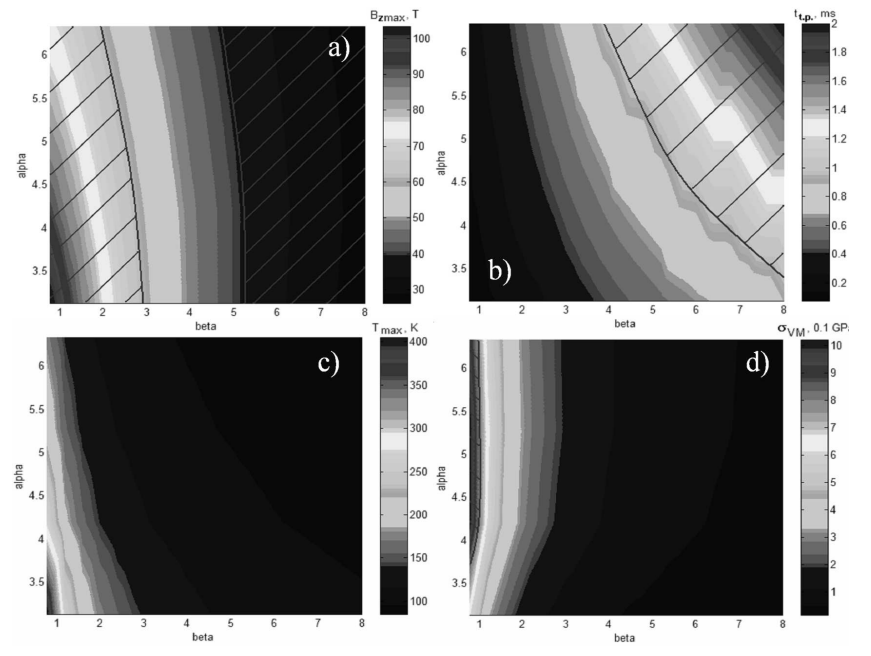

Fig. 3. (a) Magnetic field $B_{z \max }(\alpha, \beta)$, (b) pulse rise-time $t_{\mathrm{tp}}(\alpha, \beta)$, (c) winding temperature after the pulse $T_{\max }(\alpha, \beta),(\mathrm{d})$ maximum stresses $\sigma_{\mathrm{VM}}(\alpha, \beta)$.

Figure 3c indicates that using all the analyzed geometric configurations inductors were not overheated and this fact proves the success of previous modelling and experiments [5] when the material, wire and winding parameters were chosen.

Lower $\beta$ in Fig. 3d means low number of windings and low resistance of the winding subsequently and it leads to very high currents and magnetic fields, therefore stresses 
at low $\beta$ exceed $\mathrm{CuNb}$ yield strength and such inductors would be unusable although easier to construct.

After comparing discussed results inductor's "life-zone" shown in Fig. 4 is evaluated. It can be seen that an inductor would not be damaged after the pulse when $\alpha$ and $\beta$ are approximately in the range of $(3,5)$. This means that the applicable inductor windings per layer number $n_{1}$ would be in the range of $(8,12)$ and layer number $n_{2}$ would be $2,4,6$. Finally, the optimal inductor's geometry for the winding configuration shown in Fig. 1 and described in Table would be $n_{1}=10, n_{2}=4$.

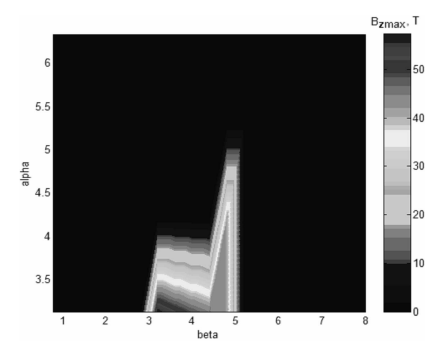

Fig. 4. Inductor's "life-zone".

Such methodology for picking up the inductor's geometry can be recommended and it can be used for any winding configuration. It is universal at solving the difficult task described in the beginning of this paper.

\section{Conclusions}

The developed techniques and models were successfully used to define inductor's "life-zone" for selected inductor winding's configuration which enables to construct such inductors less time and cost-consuming. These models can also easily be used to solve other difficult tasks like determining the "life-zone" of inductors with different wire cross-sections or materials in different layers, inductors in different environments (room temperature, $\mathrm{N}_{2}$, other usual experimental temperatures), different winding configurations and enforcements.

\section{Acknowledgments}

The work was supported by the Lithuanian Science and Studies Foundation.

\section{References}

[1] K. Rosseel, F. Herlach, W. Boon, Y. Bruynseraede, Physica B 39, 321 (2001).

[2] A. Lagutin, K. Rosseel, F. Herlach, J. Vanacken, Y. Bruynseraede, Measur. Sci. Technol. 14, 2144 (2003).

[3] J. Novickij, R. Kačianauskas, V. Filipavičius, R. Tolutis, S. Balevičius, N. Žurauskienè, Electron. Electr. Eng. 7, 56, 19 (2004).

[4] F. Herlach, N. Miura, Sci. Technol. 1, 204 (2003).

[5] J. Novickij, S. Bartkevičius, Electron. Electr. Eng. 2, 74,19 (2007) 